\title{
LAS FUENTES DE LAS LECTURAS DE A. POLICIANO EN LA EDITIO PRINCEPS DE PALADIO
}

The general opinion of Palladius' scholars is that the readings of A. Politianus in the Palladian editio princeps are conjectures. However, the collation we have presented in this paper, which as far as we know is the first one ever done, allow us to establish with certainty the manuscript sources of A. Politianus.

\section{La FAMilia $s, f, m, \varepsilon, e$.}

Desde hace algo más de un siglo la crítica ha venido calificando de conjeturas las lecturas que el humanista italiano A. Policiano había realizado en los márgenes o entre las líneas de la edición príncipe de Paladio ${ }^{1}$.

Pero, incluso sin el preciso cotejo entre la edición de Policiano y los códices que nos han hecho conocer la obra paladiana, puede ponerse en tela de juicio que Policiano se hubiese limitado a conjeturar. En efecto, desde un plano teórico, los estudiosos de la Crítica Textual habían señalado el enorme respeto que Policiano, en la tónica del humanismo renacentista, había tenido al codex peruetustus, hasta el extremo de considerar los manuscritos posteriores, copias del ejemplar antiguo ${ }^{2}$.

En este artículo trataremos de mostrar que las lecturas del citado humanista no son conjeturas, sino que Policiano tuvo acceso a un códice de Paladio de venerable antigüedad. Nos basamos para esta aseveración, en las importantes coincidencias observables entre las lecturas de Policiano y las ofrecidas por los manuscritos de Paladio que designaremos por las siglas $s$ (Cod. 1369 , s. xIr, Troyes, Bibliothèque Municipale), $f$ (Cod. Laur. Plut. 24 , sin. 6, s. xirr, Florencia, Biblioteca Medicea Laurenziana), $m$ (Cod. Ambr. B. 9I sup., s. XIII-XIv, Milán, Biblioteca Ambrosiana), \& (Cod. Vat. Lat. 2I93, s. xIv, Ciudad del

1 Iil ejemplar se encuentra en la Biblioteca Nacional de Paris, nún. Rés. S. 439 .

3 Cf. R. Sabbadini, Il metodo degli umanisti, Firenze, I92n, p. 56 ss. 
Vaticano, Biblioteca Apostólica Vaticana) y $e^{1}$ (Cod. lat. fol. 587, s. XIII segunda mitad, Berlín, Staatsbibliothek) ${ }^{2}$.

Estos manuscritos constituyen una familia ${ }^{3}$ dentro de la compleja transmisión de la obra de Paladio, como puede deducirse de las lecturas compartidas exclusivamente por ellos a lo largo del Generale Praeceptum, que nos llevan a suponer un modelo común designado por $\beta$ :

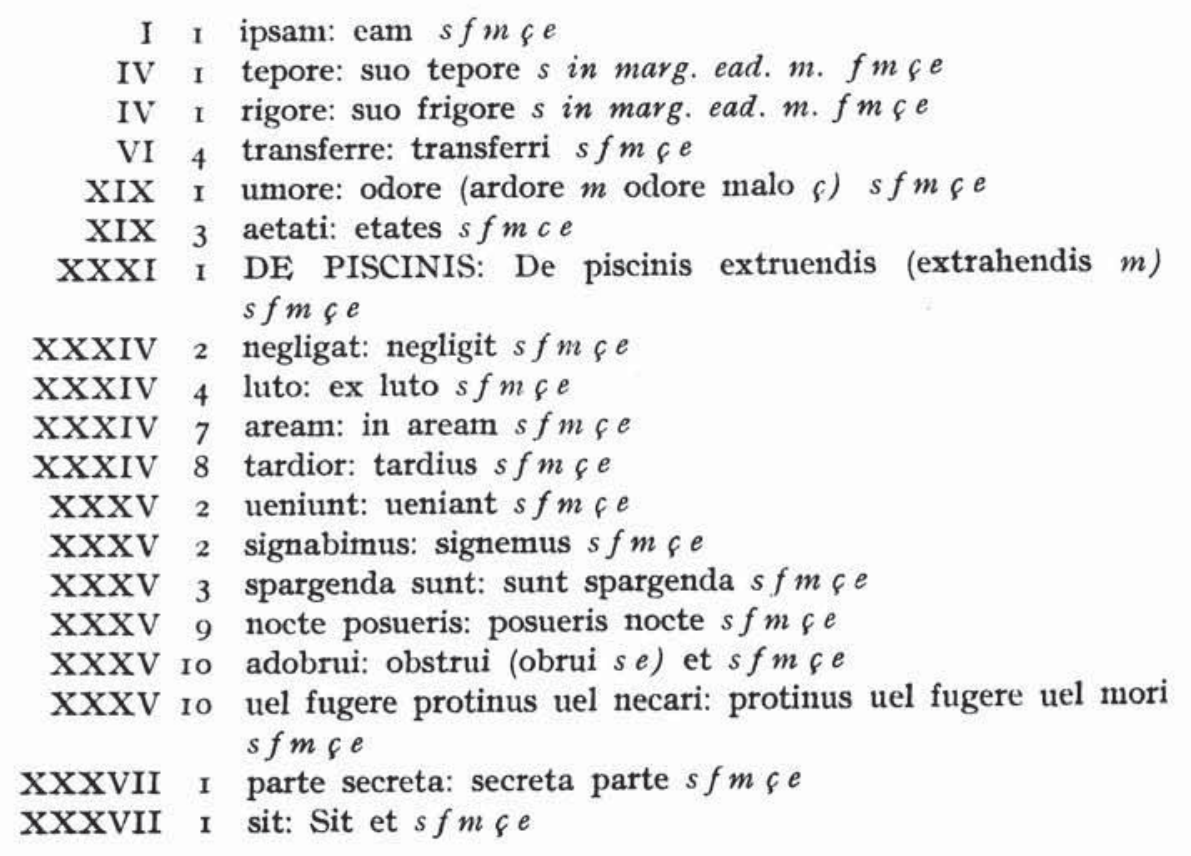

1 Este manuscrito, desconocido en los anteriores estudios y editores de $\mathrm{Pa}$ ladio, incluso también por el "Institut de Recherche et d'Histoire des Textes", llegó a nuestras manos gracias al Dr. Gerard Achten, bibliotecario de la «Staatsbibliothek» de Berlin occidental que tuvo la amabilidad de acceder a nuestra petición de un microfilm del códice.

1 De la descripción de estos códices nos hemos ocupado en nuestra tesis doctoral, Palladius liber primus. Tradición manuscrita y edición crítica, Universidad Complutense, Madrid, 1977, pp. 48, 21-22, 36-37, I4-15, respectivamente; en dichas páginas pueden verse las características de critica externa comunes a tales manuscritos. De nuestra tesis hemos tomado también una parte de los datos referentes al libro I aqui expuestos.

1 De los manuscritos que consideramos integrantes de este grupo, el códice Vat. 2193 (६) asi como las lecturas de Policiano (Pol.) habian sido cotejadas y situadas en un stemma con anterioridad a nosotros por J. C. Schmitt en su edición del libro I de Paladio (Würzburg, 1876). Este manuscrito ya no fue, en cambio, llevado a su posterior edición de Teubner en 1898 .

La situación stemmatica que nosotros atribuimos a este códice también difiere de la sostenida por este autor. 
XXXVII 2 dicitur: dicitur a $s f m f e$

XXXVII 2 remoueantur inimicae: inimice remoueantur $s f m \varepsilon e$

XXXVII 3 effundit: effundet $s f m \varepsilon e$

XXXVII 7 muniri debere: debere muniri $s f m \xi e$

Las siguientes lecturas comunes registradas entre los manuscritos y la edición de Policiano, prueban la relación que defendemos. En síntexis son:

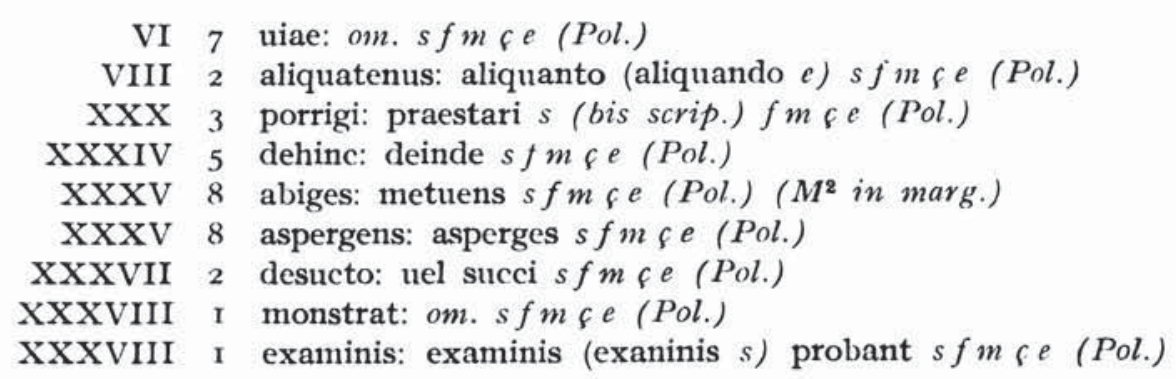

Una cala efectuada en la totalidad de la obra de Paladio nos permite exponer además las siguientes coincidencias observables entre $\beta^{1}$ y la edición de Policiano:

II 3, 3 primordio: principio $\beta$ ( Pol.)

II 9,2 sarculetur proficiet: sarculetur non affligitur sed proficiet $\beta$ (Pol.)

II ro, 3 cuestos uirga in qua pracdictae altitudinis modus designatus est per spatia quae fodientur exploret: per spatia quae fodientur custos exploret uirga in qua praedicta altitudinis modus designatus est (Pol.)

II I3, I Sed: om. $\beta$ (Pol.)

II $20 \quad$ subfundere: superfundere $\beta$ (Pol.)

II 22 redicae uel: om. $\beta$ ( $P$ ol.)

III 4 solo: loco $\beta$ (Pol.)

III I 4 coguntur: stringuntur $\beta$ (Pol.)

III I4 indigentia: indulgentia $\beta$ (Pol.)

III 17,8 suci: persici $\beta$ (Pol.)

IV 2 ueruacta: beruacita $\beta$ (Pol.)

VIII 9 olei hispani: olei Panis $\beta$ (Pol.)

$\mathrm{X} 2$ et adoreum: om. $\beta$ (Pol.)

$\mathrm{XI} \mathrm{I}_{4}$, I tantum: $\operatorname{tamen} \beta$ (Pol.)

XII 5 et ridicae: om. $\beta$ (Pol.)

1 Entiéndase por $\beta$ (según el procedimiento ya tradicional de la Crítica Textual): $\gamma=\varsigma=e ; \gamma=\varsigma ; \gamma=e ; \xi=e$. Sistema extensible a las concordancias $\gamma$ Pol. a partir de libro II. 
La necesidad de establecer un hiparquetipo $\gamma$, viene demostrada por la importancia y por el número de variantes en que coinciden de un modo totalmente exclusivo los manuscritos $s \mathrm{f}$ :

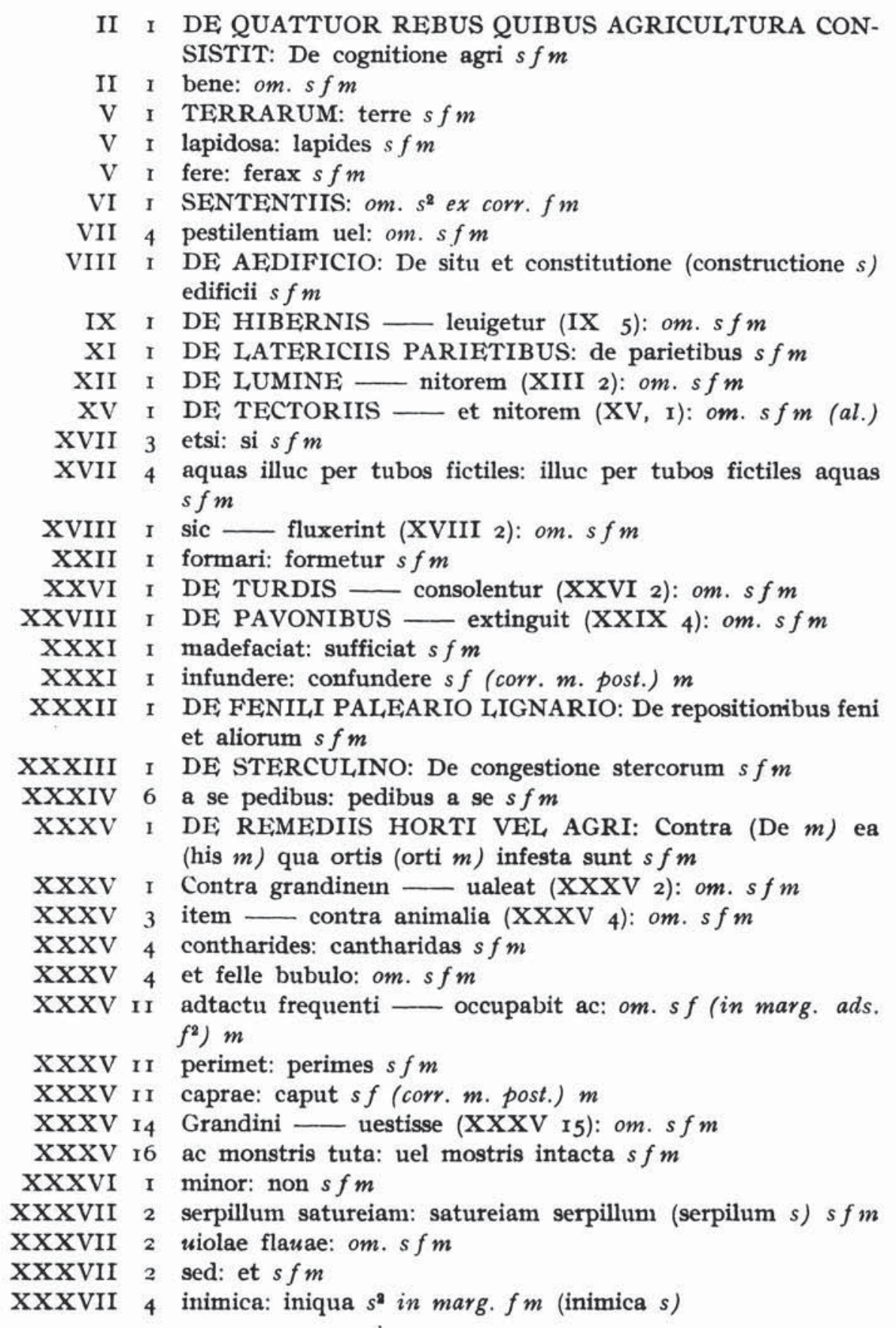


Muchas de estas variantes significativas aparecen compartidas por Policiano:

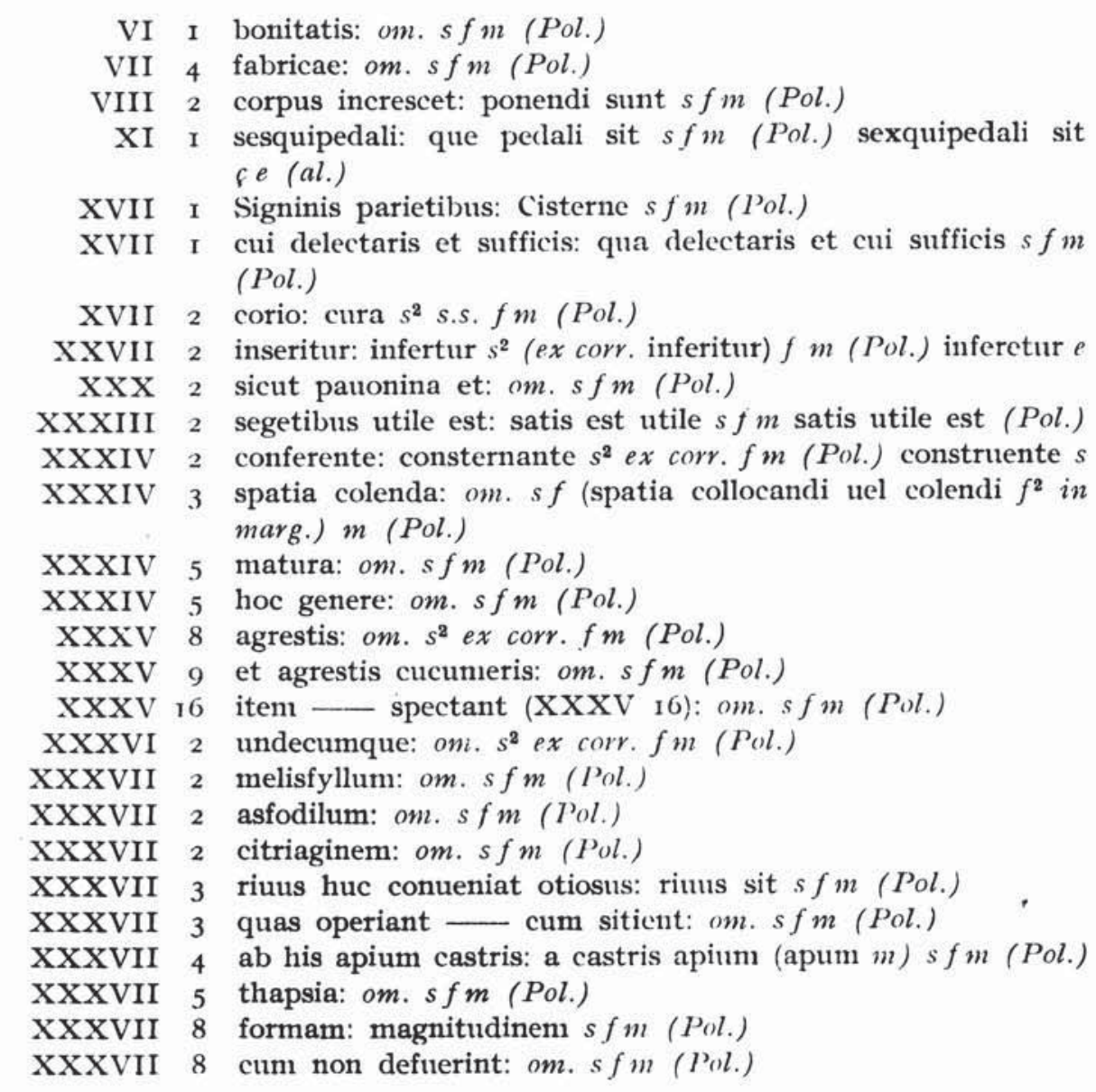

Incluso, cuando acaba el texto de $f$ en el libro primero, los dos manuscritos $s m$ siguen mostrando coincidencias, algunas de ellas comparticlas también por Policiano:

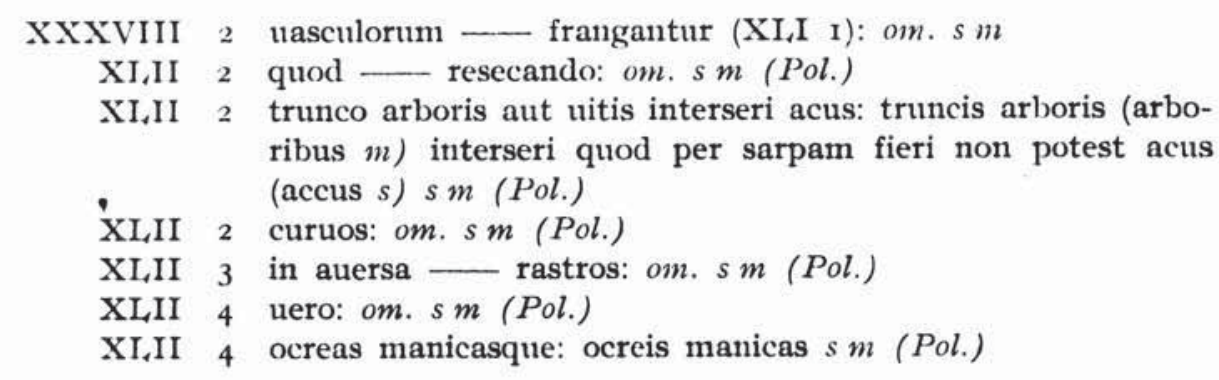


sin que las coincidencias se limiten al libro I, sino que del resto del $O p u s$ Agriculturae seleccionamos además las siguientes:

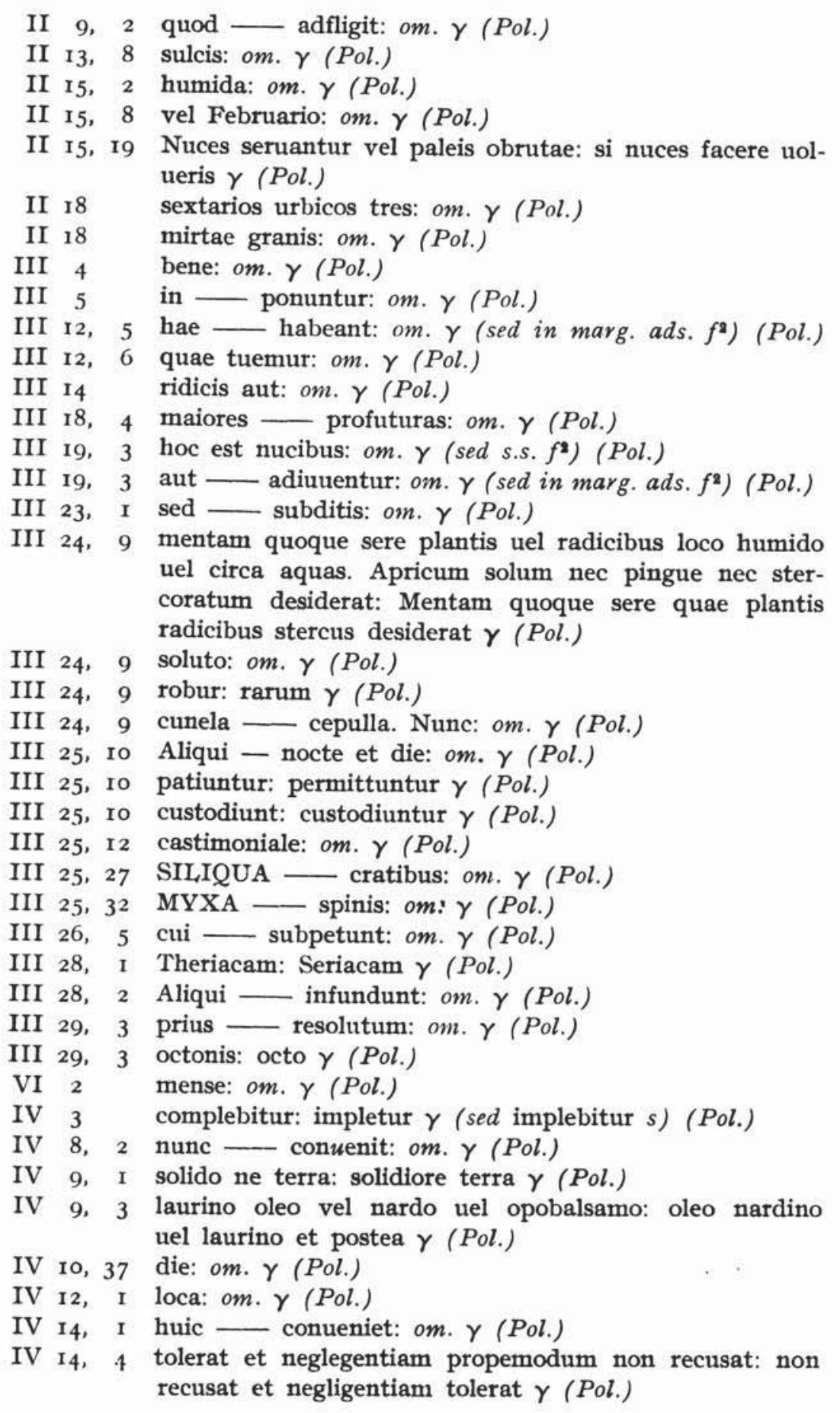




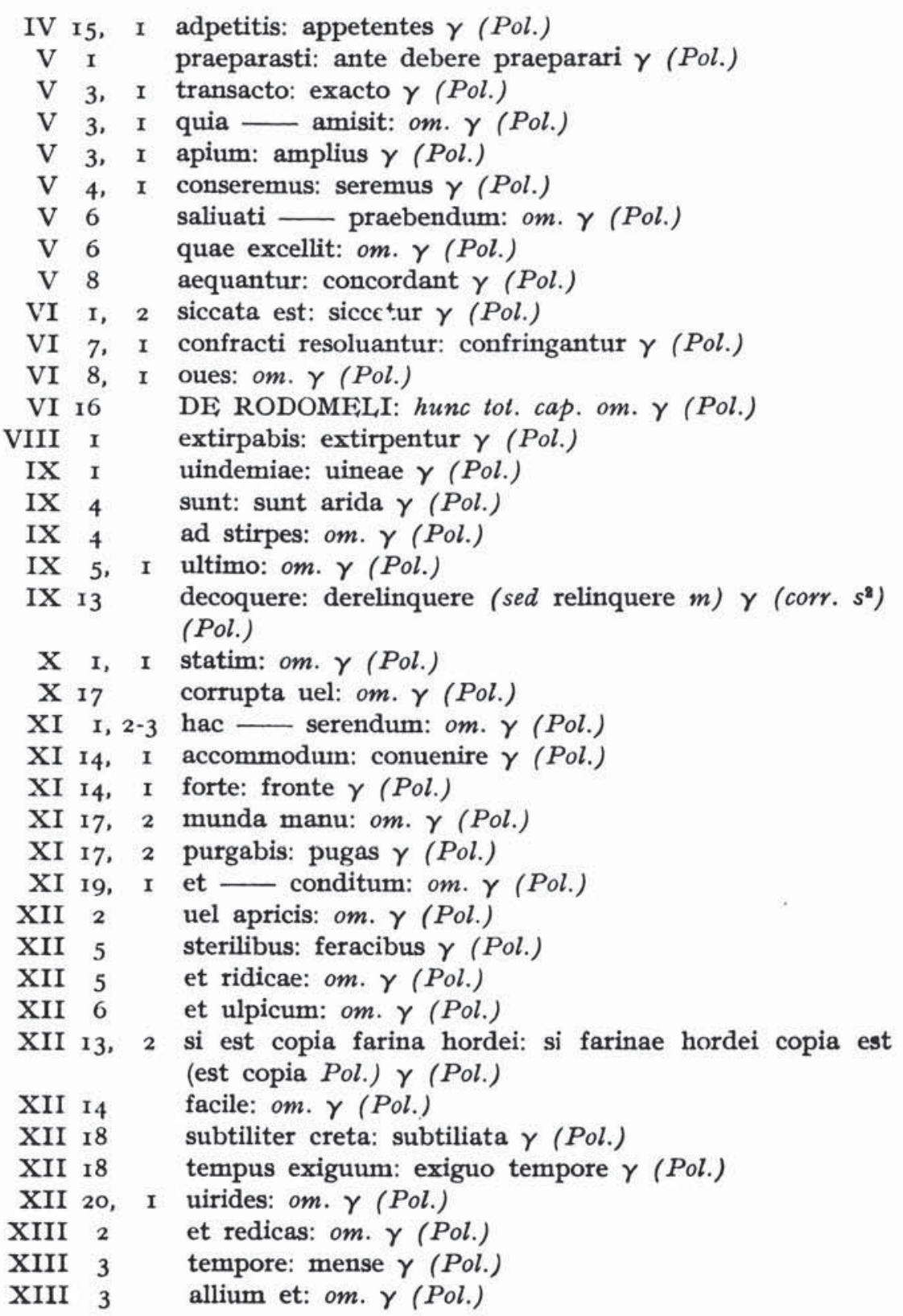

Conviene, pues, establecer las relaciones "stemmaticas" del grupo en cuestión.

Las palabras remeantis examinis probant marcan el final del texto ofrecido por el manuscrito $f$ para el libro I; sigue sin solución de continuidad mediocriter infusus ager, párrafo ya perteneciente al libro II, capítulo III 3 . 
Muestra ciertos indicios de contaminación por la presencia de dobles lecturas:

III I exercent: emittunt $f$ (exercent s.s. $\left.f^{2}\right)(M$ : Cod. Ambr. C. $2 \mathrm{I} 2$ inf.)

Por otra parte, of rece coincidencias por separado con $s$ y $m$.

Así, con $s$ :

INCIPIT TITULI: Incipit praefatio $s f$

VI 9 sarmenti: argenti $s f$

XXXV 9 in exitum: mixtum $s f$

XXXVIII I DE APIBUS EMENDIS: De apibus emendis et qualiter custodiantur $s f$

Del mismo modo, comparte con $m$ las siguientes:

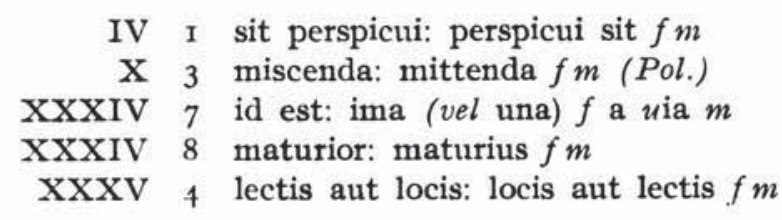

Aparte de estos indicios de contaminación, es evidente que ninguno de los manuscritos de este grupo deriva de otro: la fusión del libro I y II en $f$, fenómeno que no ocurre ni en $s$ ni en $m$, imposibilita que $s$ o $m$ hayan sido copiados de él y, por otra parte, las variantes comunes, antes citadas, entre $s f$ de las que, en cambio, no participa $m$, indican que tampoco $m$ puede derivar de ninguno de los dos. La datación de $m$ impide otras posibles alternativas.

\section{Dos manuscritos contaminados: $q e$.}

No obstante las variantes que comparten estos manuscritos con $s f m$, sin embargo se revelan como testimonios contaminados, por el hecho de tener variantes significativas, comunes con manuscritos de otras ramas.

Tal es el caso de $e$ que coincide, independientemente de $f$, con $s f m$, en los siguientes lugares:

I 2 facere uel nutrire: nutrire uel facere $s f m e$

I 2 cum sua omni: omnia cum sua $s f m e$ 
IV I ducatur: deducantur $s f m e$

IV 2 quae supra sunt corrupta dimittunt: demittunt que supra sunt corrupta $s$ f $m e$

$V$ I quaerenda fecunditas: fecunditas querenda $s f m e$

$\mathrm{V} 2$ ferat: om. $s f m e$

VI I haec erit: erit haec $s f m e$ (Pol.)

VI I in primis debeas: debeas in primis $s f m e$

VI 2 genera: post frugum scrip. $s f m e$

VI 5 debet: terra (tamen $s e$ ) iubetur $s$ ex corr. $f m e$

VII 4 animalia inimica: inimica animalia $s f m e$

$\mathrm{XI} 1$ possint penetrare perpluuia: possit pluuia penetrare $s f m e$ (Pol.)

$\mathrm{XI}$ I eos prius: prius eos $s f m e$

XIV I calcem: om. $s$ fme

XVII 4 ad bibendum omnibus antefertur: omnibus antefertur ad bibendum sf $m e$ ( $W$ : Cod. Iieja $77 \mathrm{~B}$ )

XVII 4 debeat et hortorum: et ortorum debeat $s \mathrm{fme}$

$\mathrm{XIX} I$ DE HORREO: de situ horreorum $s$ f $m e$

$\mathrm{XX}$ I munita: obmunita $s \mathrm{f} e$

XXIV I sublimis una: sublimissima $s \mathrm{fm} e$

XXIV 3 fabam vel eruum: uel herbum fabam $s f m e$

XXIV 3 tritici sufficient: sufficiunt tritici $s$ f $e$ (Pol.)

XXIV 3 mensibus praebeamus hibernis: praebeamus hibernis mensibus $s$ f $m e$

$\mathrm{XXV}$ I maceratum semper: semper maceratum $s f m e$

$\mathrm{XXV} I$ frequenter mundior: mundior frequenter $s f m e$

XXVII I his: eis $s$ f $m e$

XXXIII 2 sit: est $s f m e$

XXXIII 2 proficient: proderunt $s$ fme (Pol.)

XXXIV 6 funes cum seminibus obruemus: cum seminibus obruemus funes $s$ in $e$

En otros lugares $e$ es, en cambio, coincidente con otros códices:

VI 14 ager: om. $e$ (al.)

XIII I et aequales constituemus: constituemus et equales $e$ (al.)

XXVIII I plerumque per agros uagati: per agros uagati plerumque $e$ (al.)

Por su parte $f$ presenta un texto con abundantes lecturas particulares y adiciones muy notorias. Por ejemplo:

\footnotetext{
I 2 rusticis: urbånis ac rusticis \&

III I adprobatur: declarant $\&$

III I caeli illius: illius aeris siue celi $\&$

V 6 sicut supra dixi eligendus est: eligendus est autem ut praedixi $f$

VI 6 sterilitas morbus uicinus: morbus sterilitas et malus uicinus $\&$
} 
VI 12 prodeant: prosit $\varsigma$

VI 13 beneficio laetandur incendii: bene si custodiantur incendiis succurrunt $\&$

VI 14 operandam Rodgers: operandum $\&$

VIII 2 cetera: cetera uicina loca $\&$

VIII 2 deputetur: deputare debebis $\&$

$X$ I nigra possedit: optinet nigra \&

XII I sicut supra dixi: ut praediximus $\&$

XV I oportet inponi: imponemus et sic $\&$

XVIII 2 quod si cupis locum suum deputabimus is locus ad calcatorii similitudinem podiis breuibus et testaceo pauimento solidetur ut etiamsi ignorata se cupa diffuderit lacu subdito excipiantur non peritura uina quae fluxerint: Debent etiam in pauimento ad calcatorii modum testatio solida fieri procliuiori loco ut si a cupis vel doliis uina profluxerint ibidem conseruentur $\&$

XXI I suspensa sint: debent esse suspensa \&

XXVI 2 munda: nitida \&

XXVII I quae modo: dum modo non sit fatua $\&$

XXVIII 6 infantum similitudine cum illis tumentes gingiuas denticuli aperire nituntur: more infantum cum tumentes denticulos primo emittunt $f$

XXXVII 6 deterrima: pessima \&

Participa en algunos lugares, al margen de $e$, de las lecturas de $s f m$, comunes exclusivamente, por tanto, a los cuatro manuscritos:

IV 2 saluo capite: saluum caput $s f m \&$

VI 5 uites: arbores uel uites $s$ (uel uites s.s.) $f m f$

XIX 3 uelut corrupto: corrupto uelut (uelut $f m$ ) $s f m f$

XXXV 4 ex oleo: om. $s f m$ \&

$\mathrm{XXXV} 6$ putatoriae: putatoriorum $s f m f$

XXXV io sic poni: supponi $s f m \&$ (Pol.)

Pero en otros lugares $\xi$ se muestra un manuscrito contaminado, tanto con el códice $n^{1}$, como con los manuscritos $A \bar{N}^{2}$ con los que comparte, entre otras, las variantes que a continuación se citan:

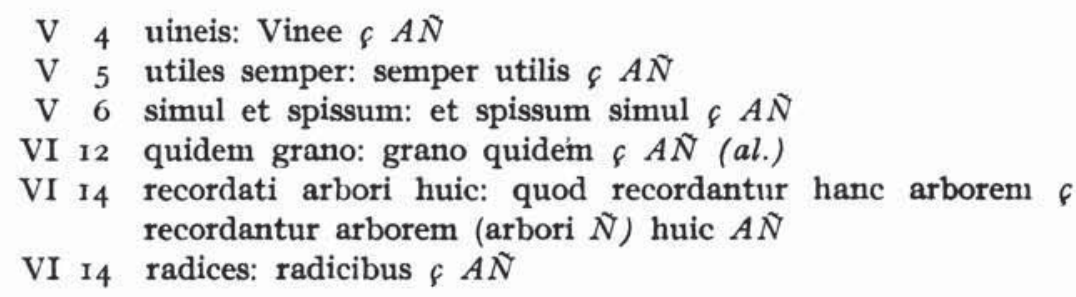

1 Cód. Cantábr. O. 3, 42 (1215 James).

2 Cód. Essc. $\&$ IV, i $(A)$ y Cód. Esc. L. III, $6(\tilde{N})$ respectivamente. 
Al mismo tiempo, $€$ no puede haber sido copiado de $e$, su contaminación es indicio de ello, pero además, ciertas variantes privativas de $e$, que no figuran en $\xi$, muestran su independencia de un modo incontrovertible:

V 6 saepe: om. $e$

VI I subieci: subiecti $e$

VI I I fossorem: fossor $e$

VI II apertus: aptus $e$

VI I4 radices: om. $e$

XIII 2 primo inpensa: inpensa primo $e$

XV I incipiet: incipimus $e$ (corr. m. post.)

XXIV 2 calciantur: calciantur fruteos uirgosus sine foliis asper $e$

XXVIII 5 a pluribus uelis: uelis a pluribus $e$

Nos encontramos, por tanto, ante manuscritos contaminados con todas las dificultades inherentes para el establecimiento de la dirección tomada por la contaminación.

En efecto, las coincidencias antes señaladas $\gamma e$ parecen requerir un stemma

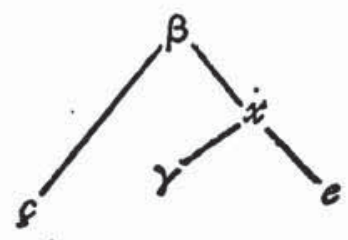

que evidentemente no daría cuenta de las lecturas comunes entre $\gamma \xi$ de las que no participa $e$, lecturas que, a su vez, tendrían que ser explicadas por un stemma opuesto al anterior:

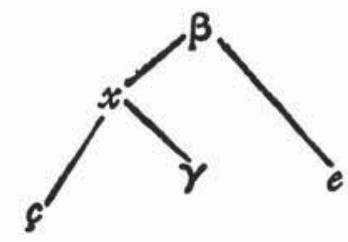

Idéntico problema es traspasable a las relaciones entre los manuscritos dependientes de $\gamma$, sin que una solución:

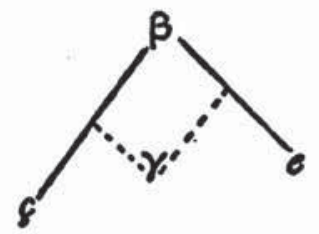


resulte tampoco plenamente satisfactoria por el señalado carácter contaminado de $\xi$ y $e$. Más probable nos parece la consideración de una contaminación originada ya en el modelo $\beta$ y multiplicada en las copias derivadas de él. Según ello, y sin pretensión de establecer un stemma maasiano, sugerimos a título de hipótesis el siguiente:

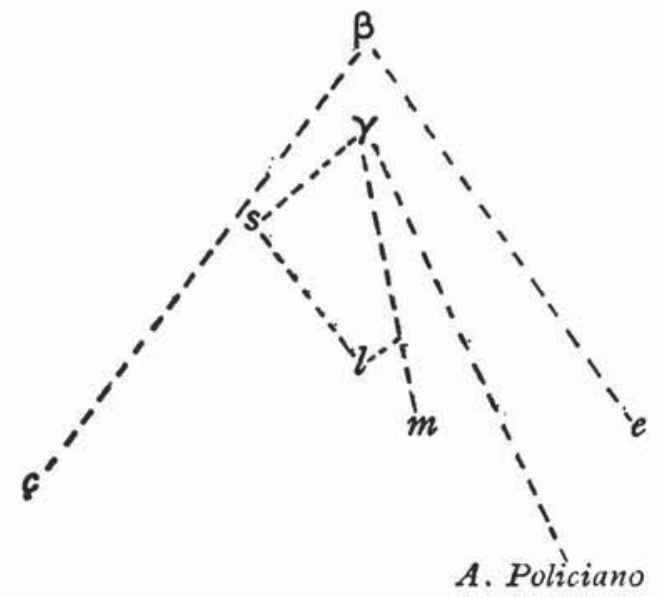

Quisiéramos añadir aquí dos notas sobre el hiparquetipo $\gamma$. La primera de ellas para señalar la coincidencia con unidades de sentido de las grandes omisiones $\gamma$; los manuscritos que dependen de tal hiparquetipo vendrían a representar, por lo menos en lo que respecta al libro I, una versión reducida de Paladio.

La segunda, para indicar que hemos conocido, por la edición de Schmitt, las lecturas que hiciera A. Policiano en la edición príncipe ${ }^{1}$.

\section{Conclusiones.}

Con esta advertencia, nos atrevemos a dar nuestra opinión personal sobre tales lecturas calificadas de conjeturas por quienes, con anterioridad a nosotros, se ocuparon de este tema.

Rodgers, por ejemplo, no cree que el humanista italiano hubiese utilizado ninguno de los códices que actualmente se encuentran en

1 Pese a nuestra petición a la Biblioteca Nacional francesa, no hemos podido conseguir el material fotográfico que hubiésemos precisado. 
Florencia y parece inclinarse por el supuesto de que las lecturas de Policiano sean en realidad conjeturas de humanistas ${ }^{1}$.

En una línea muy similar R. Martín, en su edición de los libros I y II de Paladio de muy reciente aparición, se limita a recoger la opinión de Schmitt, mencionando excepcionalmente en su aparato crítico las lecturas de Policiano sen tant que conjectures» ${ }^{2}$.

Sin embargo, muchos años antes Schmitt había plasmado en el stemma de su tesis doctoral la relación que tales lecturas guardaban con el manuscrito de la Biblioteca Vaticana 2 I93 que él designó $R$ ( $\varsigma$ para nosotros):

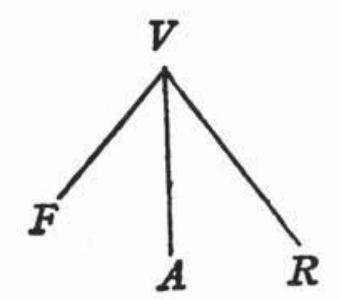

Por nuestra parte estamos más alejados de la opinión de Rodgers y Martín que de la formulada por Schmitt.

En las páginas anteriores hemos podido comprobar que las lecturas de Policiano coinciden prácticamente de un modo exclusivo con los manuscritos $s$ (siglo xiI) $f$ (siglo xIII) $m$ (siglos xir/ $/$ IIv) y naturalmente también son coincidentes con $\varepsilon e$ ocasionalmente, y sobre todo cuando estos códices contaminados comparten las lecturas de $\gamma$.

1 Cum inter doctos iam satis constet Politiani adnotationes multum in Catonem ualere eumque in Columella usum esse codice pretiosissimo (Ambrosianum dico I, 85 sup.), Politiani exemplar... diligenter contuli. Sed ex quo codice hauserit inuenire non potui; nullo enim ex eis qui nunc Florentiae adseruantur usus esse uidetur, codicem uero, quicumque is fuerit, nullius esse momenti pro certo habeo, sed lectiones nonnullas aut in apparatu notaui aut in textum recepi (e. g. 3, 9, I 2 seritate; II, 17. 3 tamen). Suspicor tamen eas nil nisi doctorum italorum coniecturas esse quas in codice aliquo saec. XIV uel XV inuenerit Politianus (Rodgers, ed. Teubneriana, 1975, pp. XV-XVI).

Su opinión es una respuesta más o menos tácita a la interpretación de Schmitt, en su edición Teubneriana de 1898: Lictiones, quas praebet $p$ i. e. Politianus, fficiunt aut codices (Florentinos), ex quibus hausit, iam interpolatos fuisse aut .b illo i.pso factas esse coniecturas... has lectiones ex editionibus Gesneri et Schneideri ddo. (J. C. Schmitt, op. cit., p. XI.)

2 R. Martin, Palladius. Traité d'Agriculture, Paris, "Les Belles Lettres", 1976, ). LIX-LX (n. 85). 
Esta comunidad de variantes habida entre las lecturas del humanista italiano y los códices (muy anteriores a Policiano) integrantes del hiparquetipo $\gamma$, creemos que resta credibilidad a quienes conjeturan que son conjeturas (valga la redundancia) las lecturas que Policiano realizó basándose, creemos, en $\gamma o$ en algún códice estrechamente emparentado con él y de respetable antigüedad, por tanto.

Si cuando se ha cumplido un siglo desde la formulación de Schmitt, no podemos mantener hoy la procedencia de ninguno de estos tres manuscritos del códice de Viena $\mathrm{I}_{4} 8(\mathrm{~V})^{\mathbf{1}}$, y si nuestra adición de tres nuevos testimonios para los que hemos supuesto el hiparquetipo $\gamma$ nos ha permitido reconstruir o, por lo menos, acercarnos a la reconstrucción de las fuentes de Policiano, a Schmitt se debe el acierto parcial $^{2}$ de haber plasmado la mayor proximidad entre el Cod. Vat. 2193 y las lecturas del conocido humanista italiano.

Ana María Moure Casas

1 Las variantes privativas de $V$ de las que carecen tanto \& como $F$ (Laur. Plut. 47, 33), pueden verse en pp. 288-289 de nuestra tesis doctoral.

1 Puede advertirse por las listas de variantes comunes del L. I antes citadas, que $f$ es el manuscrito con el que menos vinculación tienen las lecturas de Policiano, como se ha tratado de reflejar en el stemma que proponemos. 